\title{
Sentence Type Based Reordering Model for Statistical Machine Translation
}

\author{
Jiajun Zhang, Chengqing Zong, Shoushan Li \\ National Laboratory of Pattern Recognition \\ Institute of Automation, Chinese Academy of Sciences, Beijing 100190, China \\ \{jjzhang, cqzong, sshanli\}@nlpr.ia.ac.cn
}

\begin{abstract}
Many reordering approaches have been proposed for the statistical machine translation (SMT) system. However, the information about the type of source sentence is ignored in the previous works. In this paper, we propose a group of novel reordering models based on the source sentence type for Chinese-toEnglish translation. In our approach, an SVM-based classifier is employed to classify the given Chinese sentences into three types: special interrogative sentences, other interrogative sentences, and non-question sentences. The different reordering models are developed oriented to the different sentence types. Our experiments show that the novel reordering models have obtained an improvement of more than $2.65 \%$ in BLEU for a phrase-based spoken language translation system.
\end{abstract}

\section{Introduction}

The phrase-based translation approach has been the popular and widely used strategy to the statistical machine translation (SMT) since Och, et al. (2002) proposed the log-linear model. However, reordering is always a key issue in the decoding process. A number of models have been developed to deal with the problem of reordering. The existing reordering approaches could be divided into two categories: one is integrated into the decoder and the other is employed as a preprocessing module.

(C) 2008. Licensed under the Creative Commons Attribution-Noncommercial-Share Alike 3.0 Unported license (http://creativecommons.org/licenses/by-nc-sa/3.0/). Some rights reserved.
Many reordering methods belong to the former category. Distortion model was first employed by Koehn et al. (2003); a lexicalized reordering model was proposed by Och et al. (2004) and Koehn et al. (2005); and the formal syntax-based reordering models were proposed by Chiang (2005) and Xiong et al. (2006). It is worthy to note that little syntactic knowledge is used in the models mentioned above.

Compared to the reordering models that are integrated into the decoder, the reordering at the source side can utilize more syntactic knowledge, with the goal of adjusting the source language sentence to make its word order closer to that of the target language. The most notable models are given by Xia and McCord (2004), Collins et al. (2005), Li et al. (2007) and Wang et al. (2007). Xia and McCord (2004) parsed the source and target sides of the training data and then automatically extracted the rewriting patterns. The rewriting patterns are employed on the input source sentence to make the word order more accordant to target language. Collins et al. (2005) described an approach to reorder German in German-to-English translation. The method concentrates on the German clauses and six types of transforming rules are applied to the parsed source sentence. However, all the rules are manually built. Li et al. (2007) used a parser to get the syntactic tree of the source language sentence. In this method, a maximum entropy model is developed to determine how probable the children of a node are to be reordered. Obviously, there is also disadvantage in this method because the parsing tree is obtained by a full parser and contains too many nodes that are not involved in desired reorderings. Wang et al. (2007) discussed three categories which are considered to be the most prominent candidates for reordering in Chinese-to-English translation, including verb phrases (VPs), noun phrases (NPs), and localizer phrases (LCPs). The 
method deals with some special modifiers of VPs and NPs because they have the property that some specific modifiers appear before VPs or NPs in Chinese but occur after VPs or NPs in its English translation. We observe that all the transformation rules in this method are hard crafted. Furthermore, there are some other related works, such as Costa-jussa and Fonollosa's work (2006) and Zhang et al.'s work (2007). Costa-jussa and Fonollosa (2006) considered the source reordering as a translation task which translates the source sentence into reordered source sentence. A chunk-level reordering model was first proposed by Zhang et al. (2007).

However, all the existing models make no distinction between the different types of the source sentence. Intuitively, we have different reordering information in different sentence type. Taking Chinese special interrogative sentence as an example, there is a fixed phrase that usually occurs at the end of Chinese sentence but appears at the beginning part of its English translation. See the following Chinese to English translation:

Chinese: 你想要什么样的座位?

English: What kind of seats do you like?

Obviously, the Chinese question phrase “什 么样 的 座位 (What kind of seats)" should be put at the beginning of its English translation. However, many phrase-based systems fail to do this.

In this paper, we are interested in investigating the value of Chinese sentence types in reordering for Chinese-to-English spoken language translation. Due to the syntactic difference between Chinese and English, different sentence type provides different reordering information. A phrase-ahead model is developed to exploit and utilize the reordering information of special interrogative sentences. A phrase-back model is employed to catch and make use of the reordering information of other sentence types. However, the sentence type should be first identified by an SVM-based classifier before reordering the source sentence. The method overall is used as a preprocessing module for translation. We will introduce our method in detail later.

The remainder of this paper is organized as follows: Section 2 introduces our motivations; Section 3 gives the details on the implementation of our approach; the experiments are shown in Section 4; and the final concluding remarks are given in Section 5.

\section{Our Motivations}

In this section, before we analyze the Chineseto-English spoken language translation corpus, some definitions are given first.

\subsection{Definitions}

- Special interrogative sentence / other interrogative sentence / non-question sentence

Chinese sentence can be divided into question sentence and non-question sentence. If a Chinese question sentence is translated into the English sentence of wh-questions, the sentence is named as a Chinese special interrogative sentence; otherwise, it is called the Chinese other interrogative sentence. Figure 1-3 show some examples for the three sentence types respectively.

\section{- $\mathrm{SQP} / \mathrm{TP} / \mathrm{SP}$}

In Chinese special interrogative sentence, the question phrase is always moved ahead while it is translated into English. Correspondingly, the question phrase is named as the special question phrase (SQP). For example, the question phrase “什么样的座位 (What kind of seats)” in the example mentioned above is an SQP.

A few quantifier phrases (QPs) like “多次 (many times)”, “很多年 (many years)” in Chinese and some LCPs like “事故发生后 (after the accident happened)”, “会议 结束 前 (before the meeting ends)" together with some NPs like temporal phrases are named temporal phrase (TP) in our model. Some LCPs like “宾馆 前 (at the front of the hotel)”, “桌子 旁 (near the table)" and a few NPs like spatial phrases are called spatial phrase (SP) in our model. As PPs ${ }^{1}$, TPs and SPs are the most prominent candidates for reordering in Chinese other interrogative sentences and non-question sentences, they will be handled in the phrase-back reordering model.

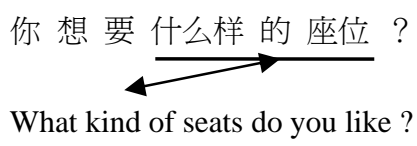

Figure 1. An example of Chinese special interrogative sentence with its English translation.

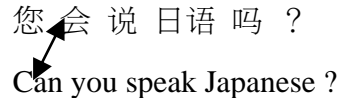

Figure 2. An example of Chinese other interrogative sentence with its English translation.

\footnotetext{
${ }^{1}$ PPs here mean prepositional phrases
} 


$$
\text { 我钱包在地铁里被偷了。 }
$$

Figure 3. An example of Chinese non-question sentence with its English translation.

\subsection{Analysis of Corpus and Our Motivations}

In order to have an overview of the distribution of the Chinese sentence types, we have made a survey based on our training set for translation, which contains about 277k Chinese and English sentence pairs. We found that about $17.2 \%$ of the sentences are special interrogative sentences, about $25.5 \%$ of sentences are other interrogative sentences and the remainders are all nonquestion sentences.

Each sentence type has its own reordering strategy, as demonstrated in Figures 1-3. There is a settled phrase (SQP) in Chinese special interrogative sentence which usually appears at the end but will be translated first in English, just as Figure 1 illustrates. For other interrogative sentences, some specific Chinese words like “会、能、可以” will just be translated into "Can" or "Do" and come first in English. At present, this information is not used in our approach. Figure 2 gives an example. For nonquestions, the reordering candidates usually need to be moved back during translation. An example is shown in Figure 3.

According to the analysis above, it is meaningful to develop reordering models based on the source sentence types.

\subsection{Framework}

As we mentioned above, our framework is illustrated as follows:

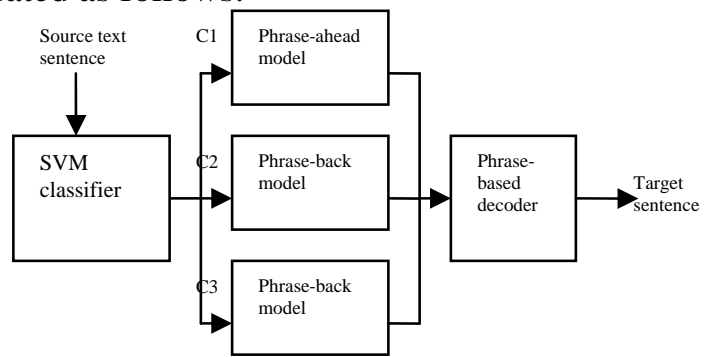

Figure 4. Architecture of the framework, where C1 means the special interrogative sentence, C2 is other interrogative sentence and C3 is nonquestion sentence.

Conventional preprocessing approaches divide the translation into two phases:

$$
S \rightarrow S^{\prime} \rightarrow T
$$

Reordering is first done in the source side which changes the source sentence $S$ into reordered one $S^{\prime}$, and then a standard phrase-based translation engine is used to translate the reordered source sentence $S$ ' into target language sentence $T$.

In our method, to utilize the information of sentence types, a new approach is proposed to improve the translation performance by developing a hybrid model as follows:

$$
S \rightarrow S^{c} \rightarrow S^{\prime} \rightarrow T
$$

Before the source sentence is reordered, an SVM-based classifier is first employed to determine its sentence type $S^{c}$, then, different reordering model is used to reorder the source sentence with the specific sentence type $S^{c}$. After getting the reordered source sentence $S$ ', we use our phrase-based SMT to obtain the optimal target language sentence.

The contribution of this paper is embodied in the first two steps of our method.

In the first step, an SVM classifier is used to identify the type of source sentence ${ }^{2}$.

In the second step, two reordering models are built according to the different sentence types. A phrase-ahead reordering model is developed for the special interrogative sentences which uses shallow parsing technology to recognize the most prominent candidates for reordering (special question phrase) and extracts reordering templates from bilingual corpus. For other sentence types, we build a phrase-back reordering model which uses shallow parsing technology to identify the phrases that are almost always moved back during translation and applies maximum entropy algorithm to determine whether we should reorder them.

\section{Models and Algorithms}

In this section, we first introduce the sentence type classifier model, and then we describe in detail the two reordering models, phrase-ahead reordering model and phrase-back reordering model.

\subsection{Sentence Type Identification}

Many models are used for classification such as Naïve Bayes, decision tree and maximum entropy. In our approach, we use an SVM-based classifier to classify the sentence types. SVM

\footnotetext{
${ }^{2}$ There are three sentence types: special interrogative sentence, other interrogative sentence and non-question sentence, which are defined in sub-section 2.1.
} 
has been shown to be highly effective at traditional text categorization. For our problem, we regard a sentence as a text. The decision boundary in SVM is a hyperplane, represented by vector $\vec{w}$, which separates the two classes, leaving the largest margin between the vectors of the two classes (Vapnik, 1998). The search of margin corresponds to a constrained optimization problem. Suppose $c_{j} \in\{1,-1\}$ (positive and negative) be the correct class of sentence $s_{j}$, the solution can be formalized as:

$$
\vec{w}:=\sum_{j} \alpha_{j} c_{j} \vec{s}_{j} \quad \alpha_{j} \geq 0
$$

Where the $\vec{s}_{j}$ is feature vector of our sentence $s_{j}$. We get $\alpha_{j}$ s through solving a dual optimization problem. Identifying the type of a sentence is just to determine which side of $\vec{w}$ 's hyperplane it will fall in.

Feature selection is an important issue. We directly use all the words occurring in the sentence as features.

Some readers may argue that the features to distinguish the sentence types are very obvious in Chinese. For example, “?” can easily separate the interrogative sentences from nonquestion sentences. In this case, a simple classifier like decision tree will work. It is true when the punctuation always appears in the sentence. However, sometimes there is no punctuation in the spoken language text. Under this situation, the decision tree will lose the most powerful features, but the performance of SVM is not affected by the punctuations. The experimental results verifying this will be given in Section4.

\subsection{Phrase-ahead Reordering Model}

As we mentioned above, about $17.2 \%$ of the spoken language sentences are special interrogative sentences. Furthermore, we note that each Chinese special interrogative sentence has one or more special question phrases (SQP) that we defined in section 2.1. Due to the difference between Chinese and English word order, the SQP needs to be moved ahead ${ }^{3}$ when it is translated into English.

Let $S$ be a Chinese special interrogative sentence, our first problem is to recognize the SQPS in $S$. If we have known the $S Q P$, namely $S$ becomes $S^{0} S Q P S^{1}$ ( $S^{0}$ is the left part of the

\footnotetext{
${ }^{3}$ There is a specific situation that the SQP don't have to be moved. In this case, we suppose it needs to be moved, but the distance is 0 .
}

sentence before $S Q P$, and $S^{1}$ is the right part of the sentence after $S Q P$ ), our second problem is to find the correct position in $S^{0}$ where $S Q P$ will be moved to.

For the first problem, because each syntactic component is possible a SQP, for example, “什 么样 的座位” in Figure 1 is NP, “在 哪里 (Where)” in Chinese sentence “我在 哪里 能 买到票 ? (Where can I buy the ticket?)” is PP (also a VP modifier), “怎么 走 (How to go)” in “去海滨怎么走? (How to go to the beach?)" is VP, it is very difficult to find the SQP by syntax. In our model, we first find out all the key words, which we list below, in the special interrogative sentences through mutual information. Then, we define the syntactic component containing the key word as an SQP. Instead of full syntactic parser, we utilize a CRF toolkit named FlexCrfs ${ }^{4}$ to train, test and predict the SQPs chunking.

\begin{tabular}{|c|c|}
\hline 什么 & What \\
\hline 哪 (哪里 / 哪儿...) & Where \\
\hline 多 (多大 /多长...) & How much/many/old... \\
\hline 怎 (怎样/怎么办...) & What about/How \\
\hline 谁 (谁的 / 是谁...) & Who/whose/whom \\
\hline 几 几点 / 几个...) & How many/old When... \\
\hline 为什么 & Why \\
\hline 何(何时 / 何地...) & When/where \\
\hline
\end{tabular}

Table 1. The special key words set

For the second problem, we note that there are only three positions where the SQP will be moved to: (1) the beginning of the sentence; (2) just after the rightmost punctuation (“,”, “;” or “:”) before the SQP; (3) or after a regular phrase such as “请问 (May I ask)” and “告诉我 (Please tell me)". Therefore, we can learn the reordering templates from bilingual corpus ${ }^{5}$. The simple algorithm is illustrated in Figure 5, and some reordering templates are shown in Table 2 .

On the whole, When we reorder the special interrogative sentence, we first identify the SQP, then we find out whether there are punctuations (“,” , “;” or “:”) before SQP; if any, we keep the rightmost punctuation index, otherwise we keep the index 0 (beginning of sentence). In the third

\footnotetext{
${ }^{4}$ See http://flexCRF.sourceforge.net

${ }^{5}$ The bilingual corpus is the corpus combined by the training corpus for chunking SQPs and its corresponding English translation.
} 
step, if we find that a reordering template like some one given in Table 2 can match the sentence, we just apply the template, otherwise we just move the SQP after the index that we kept before ( 0 or punctuation index).

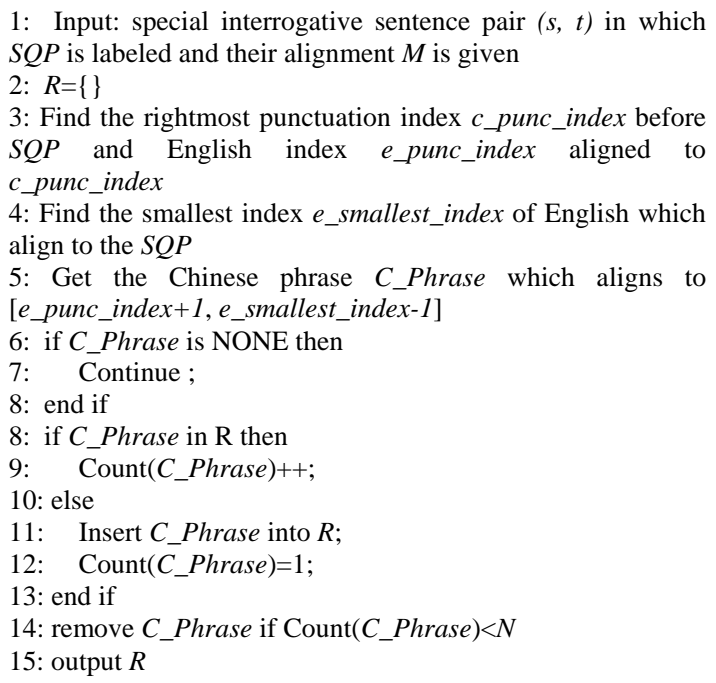

Figure 5. Reordering template extraction algorithm. The empirical value $\mathrm{N}$ is 10 in our experiment.

\begin{tabular}{|c|c|}
\hline$X 1$ 请问 $X 2 S Q P$ & $X 1$ 请问 $S Q P X 2$ \\
\hline$X 1$ 告诉 我 $X 2 S Q P$ & $X 1$ 告诉 我 $S Q P X 2$ \\
\hline$X 1$ 我想 知道 $X 2 S Q P$ & $X 1$ 我想 知道 $S Q P X 2$ \\
\hline$X 1$ 然而 $X 2 S Q P$ & $X 1$ 然而 $S Q P X 2$ \\
\hline$\ldots \ldots$ & $\ldots \ldots$ \\
\hline
\end{tabular}

Table 2. Some reordering templates

\subsection{Phrase-back Reordering Model}

In this paper, we employ the phrase-back reordering model for Chinese other interrogative sentences and non-question sentences.

Inspired by the work of Wang et al. (2007), we only consider the most prominent candidates for reordering. The VP modifiers like PP, TP, and SP which we defined in sub-section 2.1 are typically in pre-verb position in Chinese but almost always appear after the verb in its corresponding English translation. Wang et al. (2007) concentrate on VP, NP, then determine whether their modifiers should be moved back. Instead, our interests are focused on the modifiers: PP, TP and SP; namely, we consider the modifiers $\mathrm{PP}, \mathrm{TP}$ and SP as triggers, and the first VP occurring after triggers will be the candidate position where the triggers may be moved to.

Changing the focus gives us the ability to handle a specific situation that there is no VP after the triggers for recognition error or other reasons. As the example in Figure 6, there is no VP after PP (“在 这里”) because the phrase “签 名” next to PP is wrongly recognized to be a NP. To deal with the case, we will further define a fake verb phrase (FVP): the phrase after PP (TP or SP) until the punctuation (“,”, ”,” or “.”). The phrase “签名 (sign your name)" in Figure 6 is an FVP. Here, FVP is given the same function with VP, thus it makes our model suitable for more situations.

\section{请 在 这里 签名。}

Figure 6. An example of FVP. In our model the whole sentence is recognized as a VP, “在这里 (here)” is a PP, and “签名 (sign your name)” is identified as a NP.

Unlike hard reordering rules of Wang et al. (2007), we develop a probabilistic reordering model to alleviate the impact of the errors caused by the parser when recognizing PPs, TPs, SPs and VPs. We believe that no reordering is better than bad reordering. The rule forms and the probabilistic model will be given as follows:

- The form of phrase-back reordering rules:

$A: A_{1} X A_{2} \Rightarrow \begin{cases}A_{1} X A_{2} & \text { straight } \\ X A_{2} A_{1} & \text { inverted }\end{cases}$

Where, $\quad A_{1} \in\{P P, T P, S P\} \quad A_{2} \in\{V P, F V P\}$

$X \in\left\{\right.$ phrases between $A_{1}$ and $\left.A_{2}\right\}$

- We use the Maximum Entropy Model

which is implemented by Zhang ${ }^{6}$. The model is trained from bilingual spoken language corpus to determine whether $A_{1}$ should be moved after $A_{2}$. The features that we investigated include the leftmost, rightmost, and their POSs of $A_{1}$ and $A_{2}$. It leads to the following formula:

$$
P(O \mid A)=\frac{\exp \left(\sum_{i} \lambda_{i} h_{i}(O, A)\right)}{\sum_{O} \exp \left(\sum_{i} \lambda_{i} h_{i}(O, A)\right)}
$$

Where, $O \in\{$ straignt,inverted $\}, h_{i}(O, A)$ is a feature, and $\lambda_{i}$ is the weight of the feature.

When applying the rules, we first identify pairs like $\left(A_{1} X A_{2}\right)$ in the sentence, and then from beginning to end of the sentence, we move $A_{1}$ behind $A_{2}$ if $P($ inverted $\mid A)>P($ straight $\mid A)$. After all the pairs are processed, we will get the reordered source result.

${ }^{6}$ http://homepages.inf.ed.ac.uk/s0450736/maxent_too lkit.html 


\section{Experiments}

We have conducted several experiments to evaluate the models. In this section, we first introduce the corpora, and then we discuss the performance of the SVM-based classifier, chunking and reordering models respectively.

\subsection{Corpora}

We perform our experiments on Chinese-toEnglish speech translation task. The statistics of the corpus is given in Table 3 where CE_train means the Chinese-to-English training data released by IWSLT 2007; CE_sent_filtered means the bilingual sentence pairs filtered from the open resources of the bilingual sentences on the website; CE_dict_filtered means the bilingual dictionary filtered from the open resources of the bilingual dictionaries on the website; CE_dev123 denotes the bilingual sentence pairs obtained by the combination of the development data IWSLT07_CE_devset1, IWSLT07_CE_devset2 and IWSLT07_CE_devset3 which are released by the IWSLT 2007; CE_dev4 and CE_dev5 are the remainder of development data released by IWSLT 2007; CE_test means the final test set released by IWSLT 2007.

We combine the data from the top four rows as our training set. We use CE_dev4 as our development set. CE_dev5 and CE_test are our two test data. The test data released by IWSLT 2007 is based on the clean text with punctuation information, so we add the punctuation information on the Chinese sentences of CE_dev4 and CE_dev5 by our SVM sentence type classifier to form the final development set. The detailed statistics are given in Table 4.

\subsection{Classification Result}

To evaluate the performance of SVM-based classifier on classifying the sentence types, we first use a simple decision tree to divide the Chinese sentences of our training data for translation into three sentence types. Then we clean them by hand in order to remove the errors. At last, 10k sentences for each sentence type are randomly selected as the experiment data. For each sentence type, $80 \%$ of the data are used as training data, $20 \%$ as test data. Table 5 gives the classification results.

Punctuation in Table 5 means the punctuation which occurs at the end of the sentence such as “。” and "? ". We can see from the table that SVM classifier performs very well even if we remove the punctuations at the end of every sen- tence. Therefore, almost no errors will be passed to the reordering stage.

\begin{tabular}{|c|c|c|}
\hline Data & Chinese & English \\
\hline CE_train & 39,953 & 39,953 \\
\hline CE_sent_filtered & 188,282 & 188,282 \\
\hline CE_dict_filtered & 31,132 & 31,132 \\
\hline CE_dev123 & 24,192 & 24,192 \\
\hline CE_dev4 & 489 & 3,423 \\
\hline CE_dev5 & 500 & 3,500 \\
\hline CE_test & 489 & 2,934 \\
\hline
\end{tabular}

Table 3. Statistics of training data, development data and test data

\begin{tabular}{|c|c|c|c|}
\hline \multicolumn{2}{|c|}{} & Chinese & English \\
\hline \multirow{2}{*}{ Train set } & sentences & \multicolumn{2}{|c|}{276,633} \\
\cline { 2 - 4 } & words & $1,665,073$ & $1,198,984$ \\
\hline Dev set & sentences & 489 & $489 * 7$ \\
\cline { 2 - 4 } CE_dev4 & words & 6241 & 47609 \\
\hline Test set & sentences & 500 & $500 * 7$ \\
\cline { 2 - 4 } CE_dev5 & words & 6596 & 52567 \\
\hline Test set & sentences & 489 & $489 * 6$ \\
\cline { 2 - 4 } CE_test & words & 3166 & 22574 \\
\hline
\end{tabular}

Table 4. Detailed statistics of training data on development set

\begin{tabular}{|c|c|}
\hline & Accuracy (\%) \\
\hline With punctuation & 99.80 \\
\hline Without punctuation & 98.00 \\
\hline
\end{tabular}

Table 5. The accuracy of SVM classifier

\subsection{Chunking Results}

In our experiment, except that VPs are obtained by a syntactic parser (Klein and Manning, 2003), SQPs, PPs, TPs, SPs are all chunked by the FlexCrfs.

The chunking data used for training and test in Table 6 are annotated by ourselves. Every chunk is annotated according to the definition that we define in sub-section 2.1. The raw training and test data are all extracted from our training set for translation. TPs, SPs are annotated together; SQPs, PPs are annotated respectively. The statistics of the training and test data are shown in Table 6. Table 7 gives the chunking results.

The precision, recall and F-Measure are metrics for the chunking results. F-Measure follows the criteria of CoNLL-2000 ${ }^{7}$.

$$
F-\text { Measure }=\frac{2 *(\text { precision } * \text { recall })}{\text { precision }+ \text { recall }}
$$

\footnotetext{
${ }^{7}$ See http://www.cnts.ua.ac.be/conll2000/chunking/
} 
Because the SQPs have the regularity that each one contains a key word listed in Table 1, the result of SQPs chunking is quite good. Moreover, the chunking of PPs, TPs and SPs also performs well.

\begin{tabular}{|c|r|c|c|}
\hline \multicolumn{2}{|c|}{} & Train & Test \\
\hline \multirow{2}{*}{ SQP } & sentences & 10,000 & 500 \\
\cline { 2 - 4 } & chunks & 10030 & 501 \\
\hline \multirow{2}{*}{ PP } & sentences & 10,000 & 500 \\
\cline { 2 - 4 } & chunks & 10106 & 512 \\
\hline \multirow{2}{*}{ SP and TP } & sentences & 11,000 & 500 \\
\cline { 2 - 4 } & chunks & 10342 & 523 \\
\hline
\end{tabular}

Table 6. Statistics of train and test data

\begin{tabular}{|c|c|c|c|}
\hline & $\begin{array}{c}\text { Precision } \\
(\%)\end{array}$ & $\begin{array}{c}\text { Recall } \\
(\%)\end{array}$ & $\begin{array}{c}\text { F-Measure } \\
(\%)\end{array}$ \\
\hline SQP & 95.52 & 95.52 & 95.52 \\
\hline PP & 94.65 & 93.31 & 93.98 \\
\hline SP and TP & 93.92 & 92.68 & 93.25 \\
\hline
\end{tabular}

Table 7. Chunking results on test set

\subsection{Translation Results}

For the translation experiments, BLEU-4 and NIST are used as the evaluation metric. The baseline SMT uses the standard phrase-based decoder that applies the log-linear model (Och and Ney, 2002).

In the preprocessing module, all the Chinese words are segmented by the free software toolkit ICTCLAS $3.0^{8}$, and the POS tags are obtained by using the Stanford parser with its POS parsing function. For the decoder, the phrase table is obtained as described in (Koehn et al., 2005), and our 4-gram language model is trained by the open SRILM ${ }^{9}$ toolkit. It should be noted that we use monotone decoding in translation.

We have done three groups of experiments for translation. The first one is to test the effect of phrase-ahead reordering model, the result of which is shown in Table 8. Compared to the baseline system, phrase-ahead reordering model improves the results of the two test sets by $0.41 \%$ and $1.87 \%$ in BLEU respectively. The difference in the performance gains can be attributed to the fact that there are 100 Chinese special interrogative sentences in Test 2, while only 30 are found in Test 1 . Accordingly, the reordering candidates of Test 1 are much fewer than that of Test 2 . Thus, we can conclude that the more special interrogative sentences the better performance of the translation. Furthermore,

\footnotetext{
${ }^{8}$ See http://www.nlp.org.cn

${ }^{9}$ See http://www.speech.sri.com/projects/srilm
}

the results show that the reordering on special interrogative sentences is a good try.

The second experiment is conducted to test the effect of phrase-back reordering model. Table 8 gives the results. For the two test sets, the model brings an improvement to the baseline by $2.24 \%$ and $0.93 \%$ in BLEU respectively. However, the difference between them is still very big. We think there are two reasons: firstly, there are much more special interrogative sentences in Test 2 than in Test 1 , so the sentences of other sentence types in Test 2 are much fewer than that in Test 1 . Thus, fewer candidates are found in Test 2 than in Test 1 . Secondly, the average sentence length of Test 2 (6.5 words) is much shorter than that of Test 1 (13.2 words). We know that if the sentence is very short, the PP, TP, and SP will seldom occur. Naturally, only 89 candidates are found in Test 2 but 366 in Test 1. Regardless of the difference, the phrase-back reordering model indeed improves the translation quality significantly.

The last experiment merges the two reordering model together. The results in Table 8 show that the overall reordering model has done very well in both test sets: it improves the two test sets by $2.65 \%$ and $2.78 \%$ in BLEU score respectively. It demonstrates that every reordering model has a positive effect on translation. Therefore, our reordering model based on the sentence type is quite successful.

\section{Conclusions and Future Work}

In this paper, we have investigated the effect of the Chinese sentence types on reordering problem for Chinese-to-English statistical machine translation. We have succeeded in applying a phrase-ahead reordering model to process the special interrogative sentences and a phraseback reordering model to deal with other sentence types. Experiments show that our reordering model obtains a significant improvement in BLEU score on the IWSLT-07 task.

With the encouraging experimental results, we believe that we can mine more reordering information from the Chinese sentence types. In this paper, we only apply a phrase-back model to reorder Chinese other interrogative sentences. In the next step, we will try to develop a special reordering model for this sentence type. Furthermore, we plan to integrate the phrase-back model into phrase-ahead model for special interrogative sentences and investigate the value of this integration. 


\begin{tabular}{|c|c|c|c|c|c|}
\hline \multicolumn{2}{|c|}{} & BLEU (\%) & NIST & Sentences & Candidates \\
\hline \multirow{3}{*}{$\begin{array}{c}\text { Test 1 } \\
\text { CE_dev5 }\end{array}$} & Baseline & 32.16 & 6.4844 & 500 & \\
\cline { 2 - 5 } & Phrase-ahead reordering & 32.57 & 6.5579 & 30 & 31 \\
\cline { 2 - 5 } & Phrase-back reordering & 34.40 & 6.6857 & 470 & 366 \\
\cline { 2 - 5 } & Phrase-ahead+phrase-back & 34.81 & 6.7584 & 500 & 397 \\
\hline \multirow{3}{*}{ Test 2 } & Baseline & 34.04 & 5.8340 & 489 & \\
\cline { 2 - 6 } CE_test & Phrase-ahead reordering & 35.91 & 6.0693 & 100 & 97 \\
\cline { 2 - 6 } & Phrase-back reordering & 34.97 & 5.9172 & 389 & 89 \\
\cline { 2 - 6 } & Phrase-ahead+phrase-back & 36.82 & 6.1535 & 489 & 186 \\
\hline
\end{tabular}

Table 8. Statistics of translation results

Notes: candidates here mean how many candidate reordering phrases are recognized for each model. Sentences mean the number of sentences belonging to the specific sentence type, i.e. for phrase-ahead reordering in Test 1 , 31 special question phrases (SQP) are recognized in 30 Chinese special interrogative sentences.

\section{Acknowledgments}

The research work described in this paper has been partially supported by the Natural Science Foundation of China under Grant No. 60575043 and 60736014, the National High-Tech Research and Development Program (863 Program) of China under Grant No. 2006AA01Z194 and 2006AA010108, the National Key Technologies R\&D Program of China under Grant No. 2006BAH03B02, and Nokia (China) Co. Ltd as well.

\section{References}

Cao Wang, Michael Collins and Philipp Koehn. 2007. Chinese syntactic reordering for statistical machine translation. In Proceedings of joint Conference on Empirical Methods in Natural Language Processing and Computational Natural Language Learning, 2007.

Chi-Ho Li, Dongdong Zhang, Mu Li, Ming Zhou Minghui Li and Yi Guan. 2007. A probabilistic approach to syntax-based reordering for statistical machine translation. In Proceedings of $45^{\text {th }}$ Meeting of the Association for Computational Linguistics .

Dan Klein and Christopher D. manning. 2003. Accurate unlexicalized parsing. In Proceedings of $41^{\text {st }}$ Meeting of the Association for Computational Linguistics.

David Chiang. 2005. A hierarchical phrase-based model for statistical machine translation. In Proceedings of $43^{\text {rd }}$ Meeting of Association for Computational Linguistics.

Deyi Xiong, Qun Liu and Shouxun Lin. 2006. maximum entropy based phrase reordering model for statistical machine translation. In Proceedings of the joint conference of the International Committee on Computational Linguistics and the Association for Computational Linguistics 2006.

Fei Xia and Michael McCord. 2004. Improving a Statistical MT system with automatically learned rewrite patterns. In Proceedings of $20^{\text {th }}$ International Conference on Computational Linguistics.

Franz Josef Och and Hermann Ney. 2002. Discriminative training and maximum entropy models for statistical machine translation. In Proceedings of $40^{\text {th }}$ Meeting of Association for Computational Linguistics.

Franz Josef Och and Hermann Ney. 2004. The alignment template approach to statistical machine translation. Computational Linguistics, 30:417-449

Marta R. Costa-jussa and Jose A.R. Fonollosa. 2006. Statistical machine reordering. In proceedings of Conference on Empirical Methods in Natural Language Processing 2006.

Michael Collins, Philipp Koehn, and Ivona Kucerova. 2005. Clause restructuring for statistical machine translation. In proceedings of $43^{\text {rd }}$ Meeting of the Association for Computational Linguistics.

Philipp Koehn, Franz J. Och. and Daniel Marcu. 2003. Statistical Phrase-based Translation. In proceedings of HLT-NAACL 2003.

Philipp Koehn, Amittai Axelrod, Alexandra Birch Mayne, Chris Callison-Burch, Miles Osborne and David Talbot. 2005. Edinburgn System Description for the 2005 IWSLT Speech Translation Evaluation. In International Workshp on Spoken Language Translation.

Yuqi Zhang, Richard Zens and Hermann Ney. 2007. Chunk-level reordering of source language sentence with automatically learned rules for statistical machine translation. In Proceedings of SSST, NAACL-HLT 2007/AMTA Workshop on Syntax and Structure in Statistical Translation.

Vladimir Naumovich Vapnik. 1998. Statistical Learning Theory. John Wiley and Sons, Inc. 